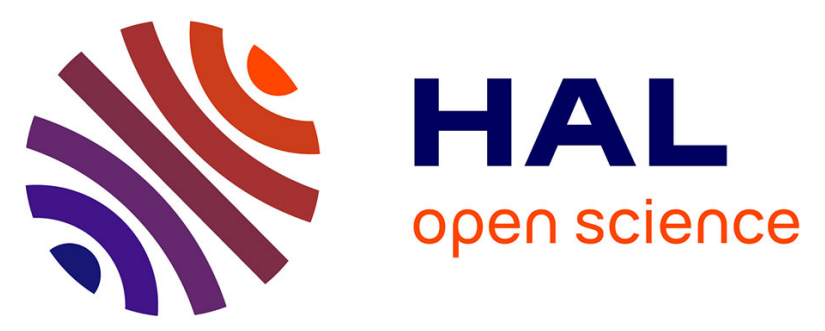

\title{
Resonant second harmonic generation in a gallium nitride two-dimensional photonic crystal on silicon
}

\author{
Y. Zeng, Iännis Roland, X. Checoury, Z. Han, M. El Kurdi, Stéphane
} Sauvage, B. Gayral, Christelle Brimont, Thierry Guillet, Meletios Mexis, et al.

\section{- To cite this version:}

Y. Zeng, Iännis Roland, X. Checoury, Z. Han, M. El Kurdi, et al.. Resonant second harmonic generation in a gallium nitride two-dimensional photonic crystal on silicon. Applied Physics Letters, 2015, 106, pp.081105. 10.1063/1.4913679 . hal-01130620

\section{HAL Id: hal-01130620 \\ https://hal.science/hal-01130620}

Submitted on 3 Jun 2021

HAL is a multi-disciplinary open access archive for the deposit and dissemination of scientific research documents, whether they are published or not. The documents may come from teaching and research institutions in France or abroad, or from public or private research centers.
L'archive ouverte pluridisciplinaire HAL, est destinée au dépôt et à la diffusion de documents scientifiques de niveau recherche, publiés ou non, émanant des établissements d'enseignement et de recherche français ou étrangers, des laboratoires publics ou privés. 


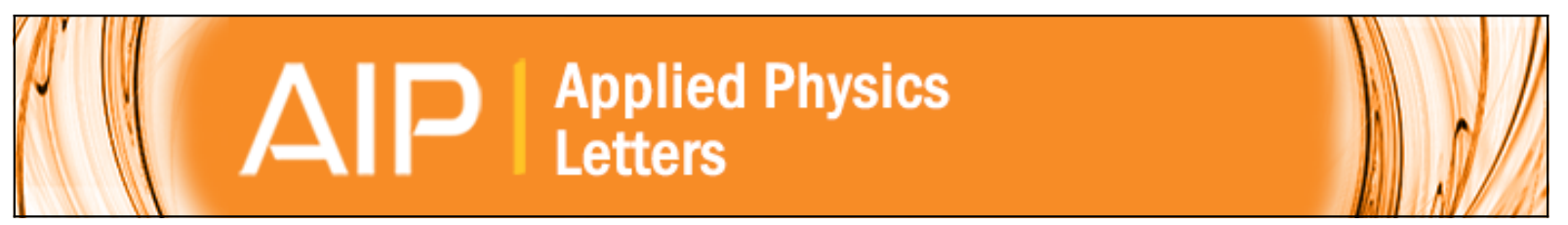

Resonant second harmonic generation in a gallium nitride two-dimensional photonic crystal on silicon

Y. Zeng, I. Roland, X. Checoury, Z. Han, M. El Kurdi, S. Sauvage, B. Gayral, C. Brimont, T. Guillet, M. Mexis, F. Semond, and P. Boucaud

Citation: Applied Physics Letters 106, 081105 (2015); doi: 10.1063/1.4913679

View online: http://dx.doi.org/10.1063/1.4913679

View Table of Contents: http://scitation.aip.org/content/aip/journal/apl/106/8?ver=pdfcov

Published by the AIP Publishing

\section{Articles you may be interested in}

Near-infrared gallium nitride two-dimensional photonic crystal platform on silicon

Appl. Phys. Lett. 105, 011104 (2014); 10.1063/1.4887065

Second harmonic generation in GaP photonic crystal waveguides

Appl. Phys. Lett. 98, 263113 (2011); 10.1063/1.3607288

Enhanced second- and third-harmonic generation and induced photoluminescence in a two-dimensional GaN photonic crystal

Appl. Phys. Lett. 87, 101106 (2005); 10.1063/1.2037849

Enhancement of visible second-harmonic generation in epitaxial GaN-based two-dimensional photonic crystal structures

Appl. Phys. Lett. 84, 1245 (2004); 10.1063/1.1649800

Scanning second-harmonic/third-harmonic generation microscopy of gallium nitride

Appl. Phys. Lett. 77, 2331 (2000); 10.1063/1.1316776

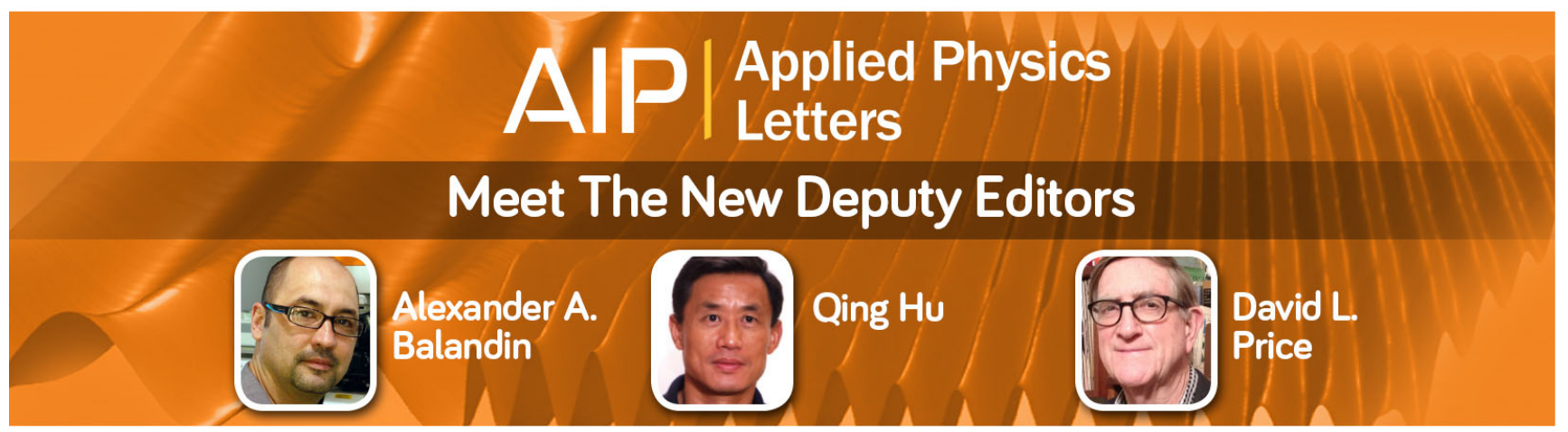




\title{
Resonant second harmonic generation in a gallium nitride two-dimensional photonic crystal on silicon
}

\author{
Y. Zeng, ${ }^{1}$ I. Roland, ${ }^{1}$ X. Checoury, ${ }^{1}$ Z. Han, ${ }^{1}$ M. El Kurdi, ${ }^{1}$ S. Sauvage,${ }^{1}$ B. Gayral, ${ }^{2,3}$ \\ C. Brimont, ${ }^{4}$ T. Guillet, ${ }^{4}$ M. Mexis, ${ }^{5}$ F. Semond,${ }^{5}$ and P. Boucaud ${ }^{1, a)}$ \\ ${ }_{1}^{1}$ Institut d'Electronique Fondamentale, CNRS - Univ. Paris Sud 11, Bâtiment 220, F-91405 Orsay, France \\ ${ }^{2}$ Univ. Grenoble Alpes, INAC-SP2M, CEA-CNRS group Nanophysique et Semiconducteurs, \\ F-38000 Grenoble, France \\ ${ }^{3}$ CEA, INAC-SP2M, CEA-CNRS group Nanophysique et Semiconducteurs, F-38000 Grenoble, France \\ ${ }^{4}$ Université Montpellier 2, Laboratoire Charles Coulomb UMR 5221, F-34905 Montpellier, France \\ ${ }^{5}$ CRHEA-CNRS, Rue Bernard Grégory, F-06560 Valbonne, France
}

(Received 27 January 2015; accepted 15 February 2015; published online 24 February 2015)

\begin{abstract}
We demonstrate second harmonic generation in a gallium nitride photonic crystal cavity embedded in a two-dimensional free-standing photonic crystal platform on silicon. The photonic crystal nanocavity is optically pumped with a continuous-wave laser at telecom wavelengths in the transparency window of the nitride material. The harmonic generation is evidenced by the spectral range of the emitted signal, the quadratic power dependence vs. input power, and the spectral dependence of second harmonic signal. The harmonic emission pattern is correlated to the harmonic polarization generated by the second-order nonlinear susceptibilities $\chi_{z x x}^{(2)}, \chi_{z y y}^{(2)}$ and the electric fields of the fundamental cavity mode. (C 2015 AIP Publishing LLC. [http://dx.doi.org/10.1063/1.4913679]
\end{abstract}

Two-dimensional photonic circuits represent key building blocks for optical signal distribution and manipulation. One of the most investigated photonic platform is based on siliconon-insulator substrates. Since silicon is an indirect band gap material, an efficient optical source with high brightness cannot be directly obtained with silicon. Moreover, silicon is a centro-symmetric material and its bulk second-order nonlinear susceptibility is equal to zero, thus ruling out electro-optic effects or frequency mixing in standard configurations. One way to circumvent these problems is the integration of III- $\mathrm{V}$ semiconductors on silicon. This can, for example, be achieved by heterogeneous integration, wafer bonding, or die bonding. Recently, photonic platforms based on nitride semiconductors have been successfully demonstrated. ${ }^{1-3}$ The nitride semiconductors benefit from several advantages. They offer a wide transparency window from the near infrared to the ultra-violet spectral range. They are recognized as the reference materials for optical sources from visible range to ultra-violet. The nitride semiconductors are non centro-symmetric and thus exhibit a second-order nonlinear susceptibility. ${ }^{4}$ By frequency doubling, it is thus possible to transfer a coherent emission to very short wavelength.

One interest of the nitride semiconductor platforms is that they can be directly integrated on a silicon substrate either by sputtering on $\mathrm{SiO}_{2}$-on-silicon ${ }^{2}$ or by direct growth of crystalline material on $\mathrm{Si}(111)$ substrates. ${ }^{5}$ The simplicity of this approach is a key advantage for the development of nitride-based on-chip photonic circuits on silicon. Twodimensional photonic crystals can also be integrated on these platforms. ${ }^{1,3,6-8}$ One specific feature of photonic crystals is the possibility to confine light in small modal volumes and with a high quality factor. This photon confinement can reinforce the nonlinear optical processes, an nth order process

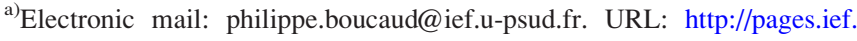
u-psud.fr/QDgroup
}

being proportional to the nth power of the ratio $\mathrm{Q} / \mathrm{V}$, where $\mathrm{Q}$ is the quality factor and $\mathrm{V}$ the modal volume. ${ }^{9-12}$

Second harmonic generation (SHG) with gallium nitride or aluminum nitride has been reported by different groups. Phase-matched conversion was observed in slab waveguides $^{13}$ or in microring resonators on oxide. ${ }^{14,15}$ Enhanced conversion was also observed in a two-dimensional GaN on sapphire photonic crystal using an out-of-plane excitation. ${ }^{16}$ In this article, we report on second harmonic generation in gallium nitride two-dimensional photonic crystal resonators. We use a free-standing two-dimensional photonic crystal platform where light is injected in the photonic crystal through suspended access waveguides. The photonic circuit is directly grown on $\mathrm{Si}(111)$. We observe second harmonic generation with a continuous wave (cw) excitation at the telecom wavelength around $1570 \mathrm{~nm}$ with less than $100 \mu \mathrm{W}$ injected power in the photonic crystal. The harmonic signal is collected at normal incidence perpendicular to the layer plane. The resonant enhancement of the harmonic conversion is evidenced. The harmonic emission pattern is modeled by finite-difference time-domain simulations and a very good agreement is found between the near-field filtered harmonic image and the one calculated from the second harmonic $\mathrm{P}_{z}$ polarization.

The studied samples were grown by molecular beam epitaxy on a $\operatorname{Si}(111)$ substrate and have been described in Ref. 3. The nitride layer consists of a thin AlN layer, around $45 \mathrm{~nm}$ thick, epitaxially grown on silicon followed by a $300 \mathrm{~nm}$ thick GaN layer. The electronic lithography of the waveguides and photonic crystal patterns was performed using a silicon oxide hard mask. The nitride material was etched using chlorinebased inductively coupled plasma ion etching. ${ }^{17}$ Dies with a total length of $500 \mu \mathrm{m}$ were cut. The silicon was then selectively under-etched using $\mathrm{XeF}_{2}$ to release the suspended structures, thus leading to free-standing waveguides and photonic crystal membranes. Light is guided in the photonic crystal by 
a W0.98 waveguide corresponding to a width of $0.98 \sqrt{3}$, where a is the photonic crystal lattice period $(580 \mathrm{~nm})$. The hole radius to period ratio was around 0.23 . The studied photonic crystal cavities were width-modulated waveguide cavities following the A1 design presented in Ref. 18. The design of these cavities consists in shifting laterally 30 holes around the cavity center with a maximum hole displacement of $12 \mathrm{~nm}$. The quality factor of the fabricated cavities is around 25000 at wavelengths close to $1550 \mathrm{~nm}^{3}$. The cavity is coupled to the access ridge waveguides by barrier coupling waveguides with a length of 17 lattice periods. cw light is injected in the photonic crystal from the sample edge through ridge waveguides suspended by nanotethers following the design presented in Ref. 19. The harmonic signal is collected perpendicular to the surface using a $\times 100$ objective with a numerical aperture of 0.9. The scattered light from the optical pump is rejected through band pass filters. Interference filters centered around the frequency-doubled pump signal were inserted in order to only image the harmonic signal. The images were taken with a photon-counting Andor electron multiplying charge coupled device (EMCCD) camera.

Figure 1 shows the superposition of an optical image of the investigated structure and the spatially resolved second harmonic signal represented in false color. Both images are taken with the same camera. The suspended access waveguides are observed on the left and right sides of the image. The two-dimensional photonic crystal length is $22.9 \mu \mathrm{m}$. Nine rows of holes are present on each side of the waveguide axis. The cavity is at the center of the photonic crystal. The light is injected from the left side with a transverse-electric polarization in the layer plane. The cavity has a quality factor of 25000 at $1577.77 \mathrm{~nm}$ and a global transmission for the photonic crystal at resonance of $-28 \mathrm{~dB}$. The second harmonic signal is obtained for the cavity pumped on resonance. A weak signal is observed on the left side after the transition between the access waveguide and the photonic crystal. The main signal is centered on the cavity and the main intensity is observed along the first two rows of the photonic crystal waveguide. A key feature is that no signal is observed at the center of the waveguide axis where the pump signal is maximum. This feature will be discussed later. The spatial extension of the second harmonic signal along the photonic crystal length is significantly larger than the one measured

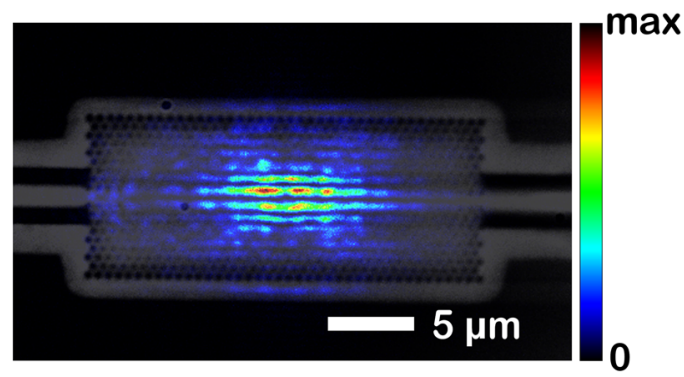

FIG. 1. Optical microscopy image of the free-standing gallium nitride photonic crystal and the second harmonic signal. The images are superimposed and recorded successively with the same camera and set-up. The widthmodulated waveguide cavity is at the center of the photonic crystal. The cavity is pumped in resonance with a cw laser at $1577 \mathrm{~nm}$. The second harmonic signal is dominated by the signal generated by the fundamental mode of the cavity. for the fundamental mode of the cavity. As the experiments are performed well below the nitride band gap, the material is transparent for both pump and harmonic. Moreover, there is no photonic band gap at the wavelength of the harmonic signal. The harmonic signal can thus propagate perpendicularly to the waveguide axis. One observes in Fig. 1 the lateral propagation of the harmonic field that is scattered by the air holes in the structure and at the interface between the photonic crystal and the non-patterned layer. We emphasize that in this experimental configuration, the harmonic signal can be analyzed with a better spatial resolution than the one for the fundamental mode at $1577 \mathrm{~nm}$ as a consequence of the larger light cone at the frequency of the harmonic signal and the better diffraction limit for the objective. We observe eight lines on each side of the waveguide axis, the spacing between the first two lines on each side of the cavity axis is around $900 \mathrm{~nm}$, and around $600 \mathrm{~nm}$ between each adjacent line. A X-cross shape with an approximate $45^{\circ}$ direction is also observed for the scattered second harmonic signal.

Figure 2 shows the second harmonic signal as a function of the input laser power at the entrance of the photonic crystal. The signal is integrated around the cavity center on a surface of $9 \times 13 \mu \mathrm{m}^{2}$. The background signal has been subtracted. One can observe a second harmonic signal with an input power in front of the photonic crystal as low as $70 \mu \mathrm{W}$. A clear quadratic dependence is observed that is confirmed by the quadratic fit of the experimental data points represented by the full line. An advantage of nitride semiconductors is their large transparency window and consequently the quenching of two-photon absorption. The two-photon absorption that can be significant in materials like silicon generally leads to a saturation of the coupled energy in the cavity and as a result to the saturation of the harmonic conversion. ${ }^{10}$ The effect is clearly not present here for the investigated input powers in the cw regime. We have estimated the experimental second harmonic conversion and collection efficiency $\eta=\frac{P_{S H G}}{P_{i n}}$, where $\mathrm{P}_{i n}$ is measured at the photonic crystal entrance and $\mathrm{P}_{S H G}$ is the collected harmonic power. The data in Fig. 2 show that the power received by the detector is $1.7 \mathrm{pW}$ for $700 \mu \mathrm{W} \mathrm{cw}$ at the crystal entrance corresponding to an effective collection efficiency of $2.4 \times 10^{-9}$. Here, the light coupling efficiency was limited by the taper

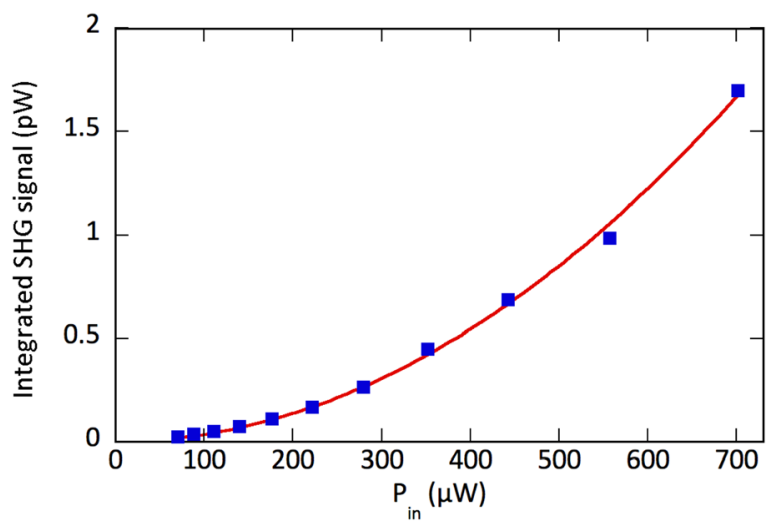

FIG. 2. Second harmonic signal integrated over the cavity center on a $9 \times 13$ $\mu \mathrm{m}^{2}$ surface as a function of the incident power at the entrance of the photonic crystal waveguide. The full line is a quadratic fit $\left(y=a x^{2}\right)$ of the experimental data. 
for this specific sample and could be increased by a factor of 5 if we refer to measurements obtained on similar structures. ${ }^{3}$ This would correspond to a cw harmonic collection efficiency of $1 \times 10^{-8}$ if we normalize by the power incident at the photonic crystal entrance. ${ }^{10,12}$ Higher conversion efficiencies have been reported by considering a power effectively coupled to the cavity. ${ }^{20,21}$ As will be discussed below, this collection efficiency corresponds to the one obtained with the signal collected perpendicular to the surface. However, the largest second harmonic polarization is along the $z$ growth axis and is not efficiently radiated towards the surface in the present experimental configuration. We note that this conversion efficiency is obtained for a singleresonant cavity mode. Higher conversion efficiencies are expected in pulsed regimes or for structures designed for double resonance and/or phase-matched conversion. ${ }^{14}$

Figure 3 shows a comparison between the transmission of the cavity and the spectral dependence of the second harmonic signal with pump wavelength. For the measurement of the second harmonic signal, the pump power is kept constant and the laser excitation is tuned across the resonance. The spectra have been normalized for clarity. The input power corresponds to the maximum one shown in Fig. 2. For the transmission, the spectrum has some asymmetry as a consequence of the incident power and thermal drift. A Lorentzian fit of the transmission resonance gives a quality factor of 25000 for this input power corresponding to a full width at half maximum of $70 \mathrm{pm}$. As a consequence of the quadratic dependence of the second harmonic signal vs. pump power, the second harmonic has a narrower line shape (40 pm full width at half maximum). This feature is a direct signature of a second order nonlinear process. It is well reproduced by the squared Lorentzian as reported in Fig. 3. The 9 pm spectral shift of the resonance between the second harmonic signal and the transmission is attributed to a temperature drift due to a much slower acquisition for the harmonic signal.

In order to interprete the image of the collected second harmonic, we have calculated the second harmonic

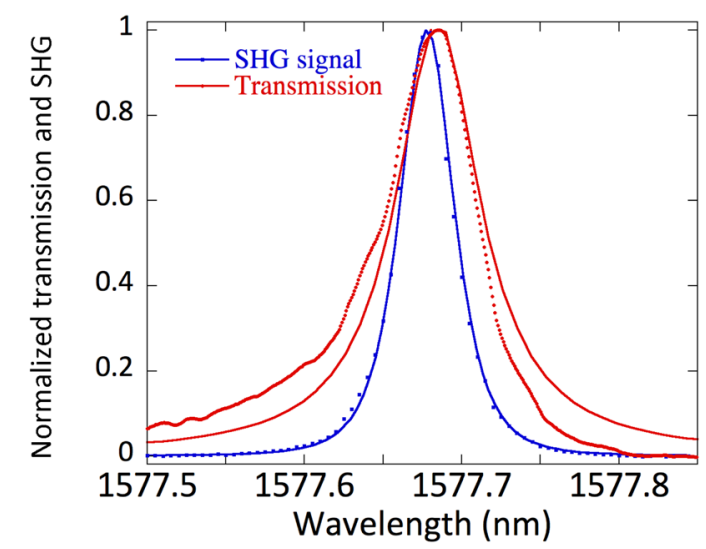

FIG. 3. Normalized transmission of the cavity (red dots) compared to the spectral dependence of the second harmonic signal (blue squares). The pump power is kept fixed. The full lines correspond to a Lorentzian fit and a squared Lorentzian fit for the transmission and second harmonic signal, respectively. The full width at half maximum is $70 \mathrm{pm}$ for the pump while the full width at half maximum of the harmonic signal is $40 \mathrm{pm}$. The linewidth reduction is a consequence of the second-order process. polarization that is generated by the fundamental mode of the cavity. The fundamental mode profile was obtained by three-dimensional finite difference in time domain modeling. For the harmonic polarization calculation $\left(P_{i}^{(2 \omega)}=\right.$ $\left.\frac{1}{2} \varepsilon_{0} \chi_{i j k}^{(2)} E_{j} E_{k}\right)$ where $E$ is the cavity mode electric field, one has to consider the different orientations of second-order susceptibility for the nitride material. The $x$ axis is along the waveguide axis, the $y$ axis is perpendicular to the waveguide, and the $z$ axis is along the growth direction. The five components of interest for the susceptibility tensor are $\chi_{z x x}^{(2)}, \chi_{z y y}^{(2)}$, $\chi_{y z y}^{(2)}, \chi_{x z x}^{(2)}$, and $\chi_{z z z}^{(2)} \cdot{ }^{22,23}$ The susceptibilities have similar amplitudes around $5 \mathrm{pm} / \mathrm{V} .^{14,23-25}$ The largest induced polarization is $\mathrm{P}_{z}^{(2 \omega)}$, i.e., the one generated by the $\mathrm{E}_{x}$ and $\mathrm{E}_{y}$ components of the confined mode. This polarization is maximum at the waveguide center in agreement with the spatial profile of the confined fundamental mode. The most interesting feature is the calculation of the propagating second harmonic field in the structure. This is obtained by injecting the $\mathrm{P}_{z}^{(2 \omega)}$ polarization in the finite-difference time-domain modeling, calculating the different components of the electric and magnetic fields, doing a Fourier transform, spatially filtering the result by the objective numerical aperture and doing an inverse Fourier transform. The Poynting vector calculated from these near-field filtered patterns are then timeaveraged. The result of this modeling is shown in Fig. 4 and compared to the collected harmonic signal as shown in Fig. 1. The main experimental features are well reproduced by the modeling. The harmonic signal is generated by the mode-localized polarization oriented along the $z$ direction. This elongated source along the waveguide axis propagates in the layer plane perpendicularly to the waveguide axis. Only a very small fraction of signal propagates along the waveguide axis, as observed experimentally. The longitudinal spatial extension of the harmonic signal is also in agreement with the experimental one. The signal vanishes at the center of the waveguide as a result of the symmetry of the harmonic electromagnetic fields. The signal is maximum at the edge of the first photonic crystal rows, as observed experimentally. One observes a series of lines parallel to the cavity axis. As the nitride material is transparent at the second

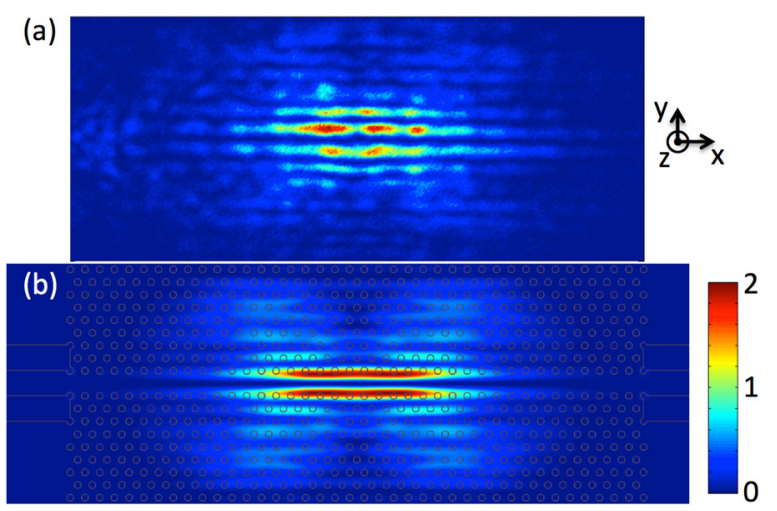

FIG. 4. (a) Zoom of the measured emission pattern of the harmonic signal as shown in Fig. 1. The full area has the dimensions of the photonic crystal structure. (b) Calculated time-averaged Poynting vector of the second harmonic signal obtained from the $\mathrm{P}_{z}^{(2 \omega)}$ second harmonic polarization generated by the fundamental cavity mode obtained by 3D-FDTD. The near-field image is filtered by the aperture of the objective. The lattice period is $580 \mathrm{~nm}$. 
harmonic wavelength, the propagation in the structure can be observed, that is not the case for structures in silicon or GaAs at similar wavelengths. This propagation leads to the $\mathrm{X}$-cross shape, i.e., less signal at the crystal center when moving to the photonic crystal edges along the $y$ axis, as a result of the spatial extension and interferences of the extended dipole sources. We emphasize that a much higher spatial resolution is obtained in these experiments as compared to the previous results on harmonic generation in $\mathrm{SiC}$ photonic crystals. ${ }^{12}$ This allows us to clearly observe the vanishing of the signal at the waveguide center that was numerically predicted in SiC but not experimentally observed. ${ }^{12}$ We have also experimentally analyzed the polarization of the emitted signal (not shown). The harmonic signal is dominated by its component with an electric field along the $y$ axis. The ratio of amplitudes between both polarizations is equal to 3.73 when integrating around the cavity center in excellent agreement with the modeling (3.85).

In conclusion, we have observed second harmonic generation in gallium nitride resonant photonic crystal cavities. The second harmonic signal was observed with a high spatial resolution allowing one to correlate the emitted pattern with the calculated near-field electromagnetic distribution. This can be achieved because of the transparency of the nitride material for both pump and harmonic. We expect to extend the properties of this photonic platform to shorter wavelengths by downsizing the photonic crystal parameters. ${ }^{17}$

This work was supported by Agence Nationale de la Recherche under QUANONIC convention (No. ANR 2013BS10-0010-03). This work was also partly supported by the RENATECH network. We acknowledge support from GANEX (Grant No. ANR-11-LABX-0014) and Labex "Nanosaclay." GANEX belongs to the public funded Investissements d'Avenir program managed by the French National Research Agency. We thank Jean Yves Duboz and Marc de Micheli for fruitful discussions.

${ }^{1}$ U. Dharanipathy, N. Vico Trivino, C. Yan, Z. Diao, J.-F. Carlin, N. Grandjean, and R. Houdre, Opt. Lett. 37, 4588 (2012).

${ }^{2}$ C. Xiong, W. H. P. Pernice, and H. X. Tang, Nano Lett. 12, 3562 (2012).
${ }^{3}$ I. Roland, Y. Zeng, Z. Han, X. Checoury, C. Blin, M. El Kurdi, A. Ghrib, S. Sauvage, B. Gayral, C. Brimont, T. Guillet, F. Semond, and P. Boucaud, Appl. Phys. Lett. 105, 011104 (2014).

${ }^{4}$ Y. Fujii, S. Yoshida, S. Misawa, S. Maekawa, and T. Sakudo, Appl. Phys. Lett. 31, 815-816 (1977).

${ }^{5}$ D. Néel, S. Sergent, M. Mexis, D. Sam-Giao, T. Guillet, C. Brimont, T. Bretagnon, F. Semond, B. Gayral, S. David, X. Checoury, and P. Boucaud, Appl. Phys. Lett. 98, 261106 (2011).

${ }^{6}$ N. Vico Trivino, U. Dharanipathy, J.-F. Carlin, Z. Diao, R. Houdre, and N. Grandjean, Appl. Phys. Lett. 102, 081120 (2013).

${ }^{7}$ D. Néel, I. Roland, X. Checoury, M. El Kurdi, S. Sauvage, C. Brimont, T. Guillet, B. Gayral, F. Semond, and P. Boucaud, Adv. Nat. Sci.: Nanosci. Nanotechnol. 5, 023001 (2014).

${ }^{8}$ N. Vico Triviño, M. Minkov, G. Urbinati, M. Galli, J.-F. Carlin, R. Butté, V. Savona, and N. Grandjean, Appl. Phys. Lett. 105, 231119 (2014).

${ }^{9}$ M. W. McCutcheon, J. F. Young, G. W. Rieger, D. Dalacu, S. Frédérick, P. J. Poole, and R. L. Williams, Phys. Rev. B 76, 245104 (2007).

${ }^{10}$ M. Galli, D. Gerace, K. Welna, T. F. Krauss, L. O’Faolain, G. Guizzetti, and L. C. Andreani, Opt. Exp. 18, 26613 (2010).

${ }^{11}$ S. Diziain, R. Geiss, M. Zilk, F. Schrempel, E.-B. Kley, A. Tunnermann, and T. Pertsch, Appl. Phys. Lett. 103, 051117 (2013).

${ }^{12}$ S. Yamada, B.-S. Song, S. Jeon, J. Upham, Y. Tanaka, T. Asano, and S. Noda, Opt. Lett. 39, 1768 (2014).

${ }^{13}$ D. N. Hahn, G. T. Kiehne, J. B. Ketterson, G. K. L. Wong, P. Kung, A. Saxler, and M. Razeghi, J. Appl. Phys. 85, 2497 (1999).

${ }^{14}$ W. H. P. Pernice, C. Xiong, C. Schuck, and H. X. Tang, Appl. Phys. Lett. 100, 091105 (2012).

${ }^{15}$ H. Jung, R. Stoll, X. Guo, D. Fischer, and H. X. Tang, Optica 1, 396 (2014).

${ }^{16}$ D. Coquillat, G. Vecchi, C. Comaschi, A. M. Malvezzi, J. Torres, and M. Le Vassor dYerville, Appl. Phys. Lett. 87, 101106 (2005).

${ }^{17}$ D. Sam-Giao, D. Néel, S. Sergent, B. Gayral, M. J. Rashid, F. Semond, J. Y. Duboz, M. Mexis, T. Guillet, C. Brimont, S. David, X. Checoury, and P. Boucaud, Appl. Phys. Lett. 100, 191104 (2012).

${ }^{18}$ E. Kuramochi, M. Notomi, S. Mitsugi, A. Shinya, T. Tanabe, and T. Watanabe, Appl. Phys. Lett. 88, 041112 (2006).

${ }^{19}$ Z. Han, X. Checoury, D. Néel, S. David, M. El Kurdi, and P. Boucaud, Opt. Commun. 283, 4387 (2010).

${ }^{20}$ K. Rivoire, Z. Lin, F. Hatami, W. T. Masselink, and J. Vučković, Opt. Exp. 17, 22609 (2009).

${ }^{21}$ S. Buckley, M. Radulaski, J. Petykiewicz, K. G. Lagoudakis, J.-H. Kang, M. Brongersma, K. Biermann, and J. Vuckovic, ACS Photonics 1, 516 (2014).

${ }^{22}$ R. W. Boyd, in Nonlinear Optics, 3rd ed., edited by R. W. Boyd (Academic Press, Burlington, 2008).

${ }^{23}$ W. E. Angerer, N. Yang, A. G. Yodh, M. A. Khan, and C. J. Sun, Phys. Rev. B 59, 2932 (1999).

${ }^{24}$ N. A. Sanford, A. V. Davydov, D. V. Tsvetkov, A. V. Dmitriev, S. Keller, U. K. Mishra, S. P. DenBaars, S. S. Park, J. Y. Han, and R. J. Molnar, J. Appl. Phys. 97, 053512 (2005).

${ }^{25}$ V. I. Gavrilenko and R. Q. Wu, Phys. Rev. B 65, 035405 (2001). 